\title{
Measuring Competitive Fitness in Dynamic Environments
}

\author{
Ivan A. Razinkov, ${ }^{\dagger, \nabla}$ Bridget L. Baumgartner, ${ }^{\dagger, \nabla}$ Matthew R. Bennett, ${ }^{\ddagger, \S}$ Lev S. Tsimring,,$\|, \perp$ \\ and Jeff Hasty ${ }^{*}, \dagger, \|, \perp, \#$
}

${ }^{\dagger}$ Department of Bioengineering, University of California San Diego, La Jolla, California 92093, United States

${ }^{\ddagger}$ Department of Biochemistry and Cell Biology, Rice University, Houston, Texas 77251, United States

${ }^{\S}$ Institute of Biosciences \& Bioengineering, Rice University, Houston, Texas 77251, United States

"BioCircuits Institute, University of California San Diego, La Jolla, California 92093, United States

${ }^{\perp}$ San Diego Center for Systems Biology, La Jolla, California 92093, United States

${ }^{\#}$ Molecular Biology Section, Division of Biological Sciences, University of California San Diego, La Jolla, California 92093, United States

Supporting Information

ABSTRACT: Most yeast genes are dispensable for optimal growth in laboratory cultures. However, this apparent lack of fitness contribution is difficult to reconcile with the theory of natural selection. Here we use stochastic modeling to show that environmental fluctuations can select for a genetic mechanism that does not affect growth in static laboratory environments. We then present a novel experimental platform for measuring the fitness levels of specific genotypes in fluctuating environments. We test this platform by monitoring a mixed culture of two yeast strains that differ in their ability to respond to changes in carbon source yet exhibit the same fitness level in static conditions. When the sugar in the growth medium was switched between galactose and glucose, the wild-type strain gained a growth advantage over the mutant strain. Interestingly, both our computational and experimental

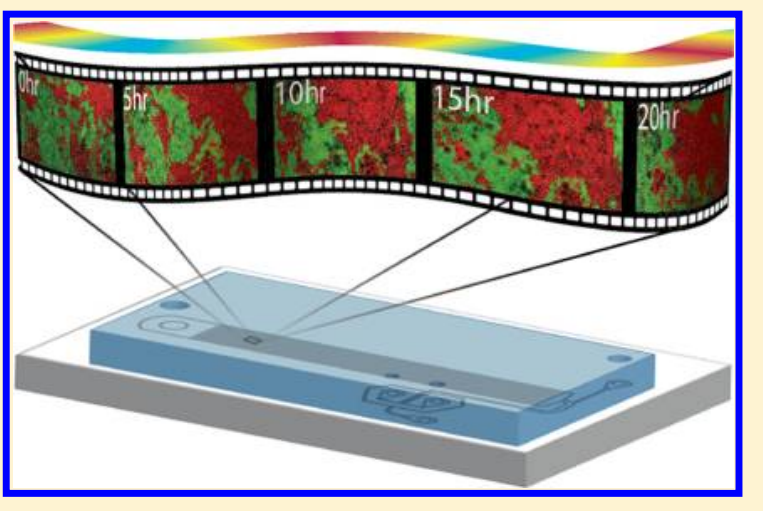
results show that the strength of the adaptive advantage conveyed by the wild-type genotype depends on the total number of carbon source switches, not on the frequency of these fluctuations. Our results illustrate the selective power of environmental fluctuations on seemingly slight phenotypic differences in cellular response dynamics and underscore the importance of dynamic processes in the evolution of species.

\section{INTRODUCTION}

The theory of evolution ${ }^{1}$ asserts that genotypes that convey optimal fitness in a given environment will be selected for through a competition for resources among individuals with various genetic sequences. The ability to predict the selective advantage of a particular allele has been exploited throughout human history in various contexts from farms to laboratories, where a specific environment is created to artificially select for desired traits. However, competitive fitness has been less well explored for cases where the selective advantage of an adaptive trait is strongly dependent on environmental dynamics. While it is easy to accept that polymorphisms that affect gene expression dynamics would influence fitness in fluctuating environments, experiments to measure actual fitness advantages in dynamic situations have been technically challenging. The goal of the present study was to invent an experimental platform for the long-term monitoring of mixed population dynamics in fluctuating environments and to use this technology to measure the selective advantage conveyed by a genetic mechanism that enhances a cell's ability to respond to environmental changes.
The ideal system for conducting dynamic evolution experiments would allow a mixed population of cells to be cocultured in a precisely controlled environment over long periods of time while granting the periodic collection of culture samples. Mixed populations can be cultured continuously in fermentors or bioreactors, but environmental dynamics cannot be precisely controlled in these devices, and the cost of replacing the medium once in every generation time can quickly become prohibitive. Microfluidic chemostats are a potentially suitable alternative because the environment can be controlled dynamically, while the small volume of the growth chamber dramatically reduces the cost of media consumption over long experiments. $^{2-5}$ However, most microfluidic devices are not designed to hold enough cells for evolution experiments and do not allow the culture to be sampled. We sought to combine the best features of large-scale fermentors and microfluidic

Special Issue: Peter G. Wolynes Festschrift

Received: March 30, 2013

Revised: July 10, 2013

Published: July 11, 2013 
chemostats to create a device that could be used to measure population dynamics. The resulting microfluidic device has a cell growth chamber large enough to maintain a log-phase culture of $\sim 10^{6}$ yeast cells for at least 500 generations. In addition, samples of culture can be collected from the effluent line for real-time monitoring of population dynamics using any of several genetic or biochemical assays. Alternatively, the monolayer growth chamber allows the experiment to be monitored by fluorescence time-lapse microscopy. The design of the media ports was based on our dial-a-wave (DAW) chip and are used to generate media fluctuations of practically any frequency or waveform, providing exquisite control over environmental dynamics. ${ }^{6}$ We named this device the evoDAW (evolution dial-a-wave) chip and tested it by monitoring the population dynamics of a mixed culture of two yeast strains that differ in their ability to respond to changes in carbon source.

Glucose is the preferred carbon source of Saccharomyces cerevisiae cells, although they are capable of growth on many types of sugar. Yeast metabolic networks contain several mechanisms to ensure that secondary carbon sources are not consumed in the presence of glucose. The most well studied of these networks is that of galactose metabolism, which is inactivated by transcriptional and post-transcriptional means in response to glucose addition. ${ }^{7,8}$ Cells growing on galactose respond to glucose by rapidly degrading specific gene transcripts encoding enzymes for galactose metabolism and entering the cell division cycle. ${ }^{7,9-12}$ The ability of galactoseinduced cells to respond to glucose has been attributed to the spatial sequestration of transcripts for galactose network genes and the cell cycle regulator, Cyclin $3(\mathrm{Cln} 3 \mathrm{p})$. The physical proximity of the transcripts creates competition for translational resources when galactose is the sole carbon source. When glucose is reintroduced, the GAL gene transcripts are specifically degraded and the competition is relieved, inducing the translation of CLN3 messages, leading to cell cycle entry. The spatial regulation of GAL1 and CLN3 transcripts is only detected in cells that have recently experienced growth in both glucose and galactose. Therefore, this is thought to be a mechanism that allows the cell to anticipate the return of glucose to the growth medium when glucose availability has been transient (Figure 1).

As part of a previous study, ${ }^{12}$ we created a mutant version of the GAL1 transcript that is stabilized in the presence of glucose. Strains expressing this allele of GAL1 (called ST for stable) degrade GAL1 messages inefficiently in response to glucose addition, and consequently the translation of CLN3 transcripts is delayed in response to glucose relative to wild-type (WT) cells. Since the cells cannot transition into the cell cycle until GAL1 mRNA has fallen below a certain threshold, small increases in the GAL1 half-life will be exacerbated on population-wide cell cycle entry delays. When growing in a constant environment containing either galactose or glucose, WT and ST cells have identical growth rates. However, the stabilization of GAL1 mRNA causes ST cells to have a transient cell cycle defect immediately following the addition of glucose to a galactose culture. We began the current study by developing a computational model to predict whether the transient difference in GAL1 mRNA levels would convey a measurable selective advantage to WT cells over ST cells growing in conditions where the sugar source alternates continuously between glucose and galactose. We then used the microfluidic evoDAW chip to experimentally validate the predictions put forth by the model. Our experimental results

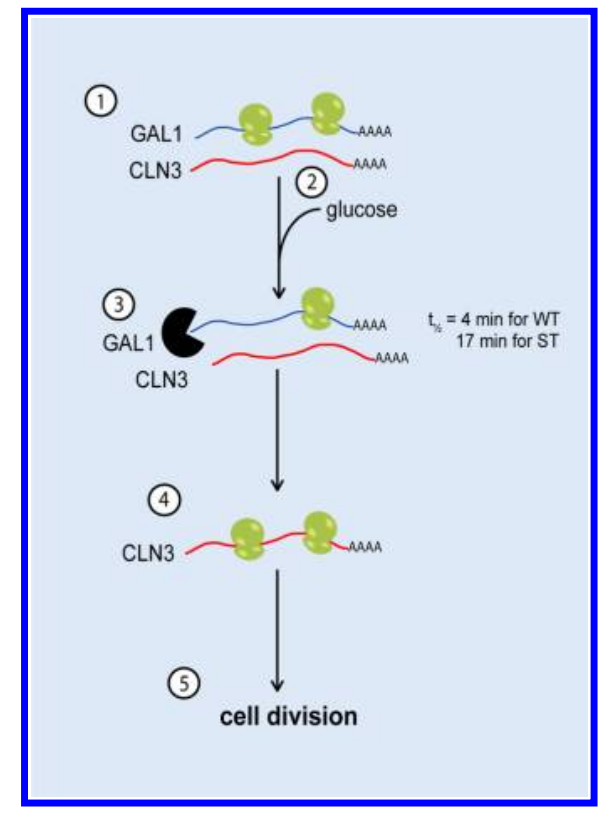

Figure 1. Translational competition mechanism regulating response of galactose-induced culture to glucose addition. (1) When cells are in galactose medium lacking glucose, GAL1 transcripts are highly expressed and spatially sequestered with less-abundant CLN3 transcripts. The GAL1 transcripts have a higher translational efficiency, so CLN3 translation is kept low, and the length of G1 phase is long. (2) Glucose is introduced into the medium triggering catabolite repression that includes the transcriptional silencing of GAL genes, and (3) the rapid degradation of GAL transcripts. The half-life of GAL1 transcripts in the presence of glucose is determined by sequences in the $5^{\prime} \mathrm{UTR}$ and is $\sim 4 \mathrm{~min}$ in the WT strain and $\sim 17 \mathrm{~min}$ in the ST strain. (4) The degradation of GAL1 transcripts relieves the competition for translation components, leading to an increase in Cln3p synthesis. (5) Cells enter S-phase as the result of $C \ln 3 p$-induced gene expression. The longer half-life of GAL1 transcripts in ST cells causes a delay in the cell cycle response to glucose.

support the model's prediction that the WT genotype does convey a selective advantage to cells growing in dynamic conditions. Interestingly, the strength of the adaptive advantage conveyed by glucose-sensitive GAL1 mRNA decay depended on the total number of carbon source switches, not on the period of these fluctuations. Our results underscore the importance of dynamic processes in the evolution of species.

\section{METHODS}

Strains. Strains were constructed as described in ref 12 . The relevant genotypes of $S$. cerevisiae strains are GAL1::LEU2GAL1 $1-1000$ to +450)-CFP-URA3 (yBB114, WT) and GAL1::TRP1-random5'UTR-GAL1(-1000 to +450)-CFPURA3 (yBB115, ST).

Experimental Section. Microfluidic evoDAW devices were assembled as previously described. ${ }^{6}$ Overnight cultures of WT and ST were grown in $25 \mathrm{~mL} 1 \%$ galactose/1\% glucose synthetic complete medium. ${ }^{12}$ The inoculated devices were then loaded with an approximately equal mixture of WT and ST cells. The microfluidic cultures were attached to two media sources: $0.5 \%$ galactose synthetic complete (medium 1) and $0.5 \%$ galactose $/ 0.25 \%$ glucose synthetic complete (medium 2 ) . The devices were incubated at $30{ }^{\circ} \mathrm{C}$ in a custom-fabricated acrylic enclosure. The media syringes were placed on automatic DAW tracks programmed to produce square wave oscillations of various periods, as previously described. ${ }^{6}$ The effluent was 
collected from the waste port (Figure 4). The devices were periodically monitored for cell growth and full occupancy using an inverted (Nikon Diaphot) light microscope equipped with $4 \mathrm{x}$ (Nikon, N.A. 0.1$)$ objective.

Data Collection and Analysis. Samples were collected every $24 \mathrm{~h}$, serially diluted 1:1000 and plated on -Leu and -Trp plates to select for WT and ST colonies, respectively. Once colonies formed, the plates were imaged using UVP BiodDoc-It Imaging System. The number of colonies in each image was counted using ImageJ. The ratio of WT cells was calculated as the number of WT colonies divided by the sum of WT and ST colonies $R=\mathrm{WT} /(\mathrm{WT}+\mathrm{ST})$. Multiple repeats were performed for each media switching period. The data for each experiment was normalized to a starting ratio of 0.5 , and average and standard error were calculated. Linear fit lines were calculated in Excel using a linear regression algorithm with $y$ intercept set to 0.5 .

\section{RESULTS}

Modeling Dynamics of Mixed WT and ST Populations. WT colonies grow better than ST colonies when the sugar source oscillates between galactose and glucose. ${ }^{12}$ The reason for this is not obvious because the differences in cell cycle dynamics between the two strains is slight and exists for only a few minutes following the transition from galactose to glucose. For most of the time, the two strains have identical growth rates. To predict whether the transient cell cycle difference provided by glucose-sensitive GAL1 mRNA could contribute significantly to population dynamics, we turned to mathematical modeling. We created a stochastic model of cellular growth and division consisting of a modified version of Gillespie's algorithm that takes into account various phases of the cell cycle (Figure2, see Supporting Information for details). We took into account the geometry of cell growth chamber and available space as a limiting factor in our model. In yeast, the restriction point beyond which a cell is committed to entering the cell cycle is called START. While in G1 phase, each cell spontaneously begins the cell cycle (i.e., passes START) with rate $r_{\mathrm{START}}$, which depends on the current carbon source and the time since the last switch. Simply put, $r_{\text {START }}$ will switch between two values, $r_{\text {gal }}$ and $r_{\text {glu }}$ for galactose and glucose environments, respectively. However, as the carbon source is changed from galactose to glucose, the switch happens according to the function

$$
r_{\text {START }}=r_{\text {glu }}-\left(r_{\text {glu }}-r_{\text {gal }}\right) \exp \left(-\lambda t^{*}\right)
$$

where $\lambda$ is the decay rate of GAL1 mRNA, and $t^{*}$ is the time since the addition of glucose. Once past START, a dividing cell enters the $S / G 2 / M$ phases. We chose to model these phases with a variable delay of average length $t_{\mathcal{c}}$ which also depends on the carbon source (Figure 2A). Since the decay rate of GAL1 mRNA differs between the WT and ST strains, so too does the rate at which $r_{\text {START }}$ relaxes after the addition of glucose. In our simulations, this difference causes the relative fraction of cells in G1 phase to decline faster in WT cells than in ST cells, similar to what was observed by flow cytometry. ${ }^{12}$ Interestingly, even though the half-lives of GAL1 mRNA while in glucose are short in both strains $(\approx 4 \mathrm{~min}$ for WT and $\approx 17 \mathrm{~min}$ for ST), the relative difference in the G1 fraction persists for much longer after the introduction of glucose. Figure $2 \mathrm{~B}$ shows the time evolution of the population fraction, $R(t)=n_{\mathrm{WT}} /\left(n_{\mathrm{WT}}+n_{\mathrm{ST}}\right)$, of a mixed population containing $n_{\mathrm{WT}}$ WT cells and $n_{\mathrm{ST}}$ ST cells

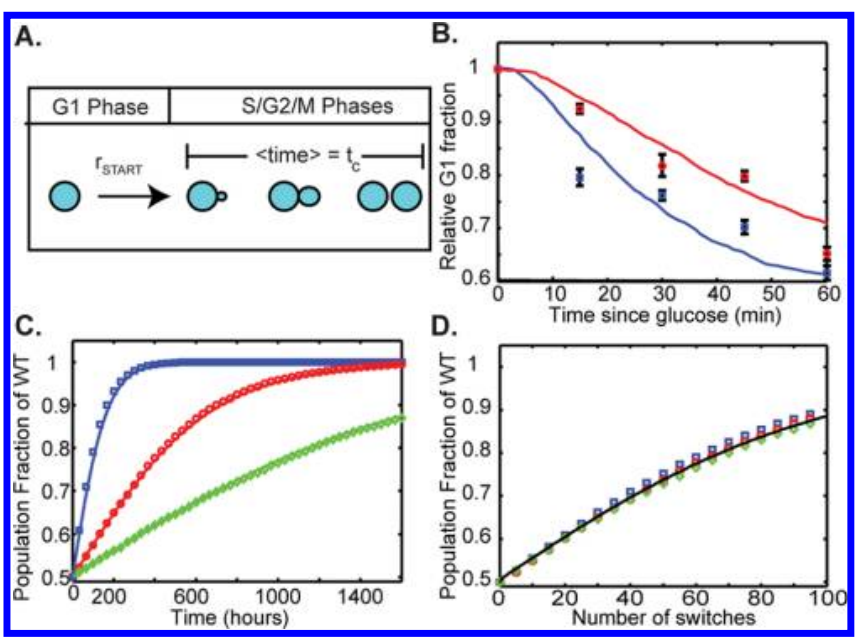

Figure 2. Modeling transcript decay-dependent growth. (A) Cellular growth in the stochastic model consists of two phases. Cells leave the G1 phase according to the rate $r_{\text {START }}$, and then enter a delayed growth and division cycle (S/G2/M phases) that takes, on average, a time $t_{c}$. (B) Relative fraction of cells in G1 phase for WT (blue) and ST cells (red) immediately after the introduction of glucose. Solid lines are the results of a single stochastic simulation and symbols are the experimental results obtained from flow cytometry. (C) Population fraction of WT in a mixed population of WT and ST cells in a dynamic environment. Symbols represent the average population fraction of 100 stochastic trials for an environmental cycling period of $100 \mathrm{~min}$ (squares), $400 \mathrm{~min}$ (circles), and $1000 \mathrm{~min}$ (diamonds). The solid line is the analytical prediction (eq 2) fit to all curves simultaneously with $\tau_{\text {eff }}=10.5 \mathrm{~min}$. (D) The same three trials plotted against the total number of switches from galactose to glucose.

in several dynamic environments, obtained from stochastic simulations. The symbols represent the averaging of 100 trials in which the media was regularly and periodically switched from galactose to glucose, and back, such that each medium was in the environment for an equal amount of time. In each simulation, the WT strain eventually takes over the entire population at a rate that depends on the period of the cycling. In particular, one can show that if the population undergoes $m$ switches from galactose to glucose, and if they remain in either environment sufficiently long, then the population fraction of the WT strain after the $m$ th switch, $R_{m}$, is given by

$$
R_{m}=\frac{1}{1+\frac{1-R_{0}}{R_{0}} \exp \left(-m \sigma \tau_{\text {eff }}\right)}
$$

where $R_{0}$ is the population fraction of the WT cells before the first switch, $\tau_{\text {eff }}$ is the effective delay in growth rate change caused by slowly decaying GAL1 mRNA, and $\sigma$ is the difference in growth rate between glucose and galactose environments (see Supporting Information for details). The solid lines shown in Figure $2 \mathrm{~B}$ are eq 2 simultaneously fit to all three trials, with $\tau_{\text {eff }}=10.5 \mathrm{~min}$ and $m=t / T$, where $T$ is the period of environmental cycling. Note that the rate of increase of the relative population fraction depends primarily on the total number of switches from glucose to galactose. If we plot each of the relative population fractions obtained from the three trials shown in Figure 2C against the total number of switches, we find that they collapse onto a single curve defined by eq 2 (see Figure 2D).

Experimental Results. To test the predictions of our computational model, we developed a novel microfluidic device capable of long-term culturing in a dynamic environment. 


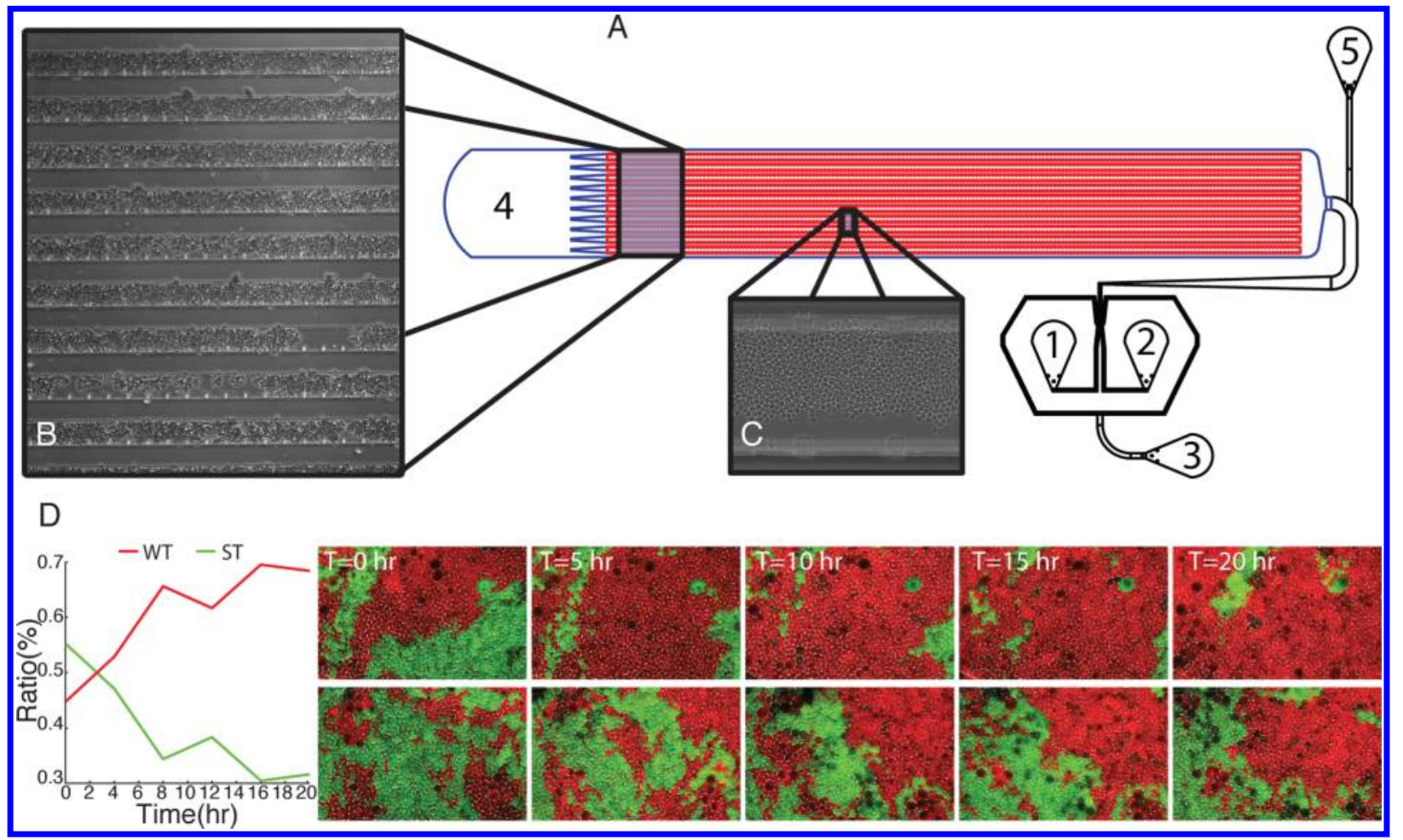

Figure 3. Overview of the evoDAW microfluidic chip design for evolutionary experiments. (A) These three ports comprise the DAW network of channels for continuous media switching. Ports 1 and 2 connect to two different media syringes, while port 3 acts as an overflow shunt for media not going to the cell chamber. Media is supplied to the cells from the DAW region and exits, along with daughter cells, through port 4 . During loading the cells enter the device through port 4 and exit through temporary port 5 . Once the cells have been loaded, port 5 is permanently filled in with silicon elastomer. (B) $4 \times$ magnification of an actual device with trapped S. cerevisiae. (C) $20 \times$ magnification. Cells fill most of the trap and maintain a uniform morphology throughout the experiment. (D) Time-lapse fluorescent microscopy images of a mixed culture of WT (red) and ST (green) cells grown in the microfluidic device. The cells were exposed to a square-wave signal of $0.25 \%$ glucose over $0.5 \%$ galactose background with $8 \mathrm{~h}$ period. Plot of ratio change as the experiment progressed. Each glucose period produced an increase in the ratio of WT cells. Time between frames is in hours. The upper and lower sets of images, while from different devices, exhibit the same take over by the WT strain.

Previous microchemostat designs, based on a mixed culture bioreactor, have the significant drawback of being unable to produce nearly instantaneous changes in the growth medium. ${ }^{13}$ We designed the evoDAW device based on a flow-through bioreactor (see Figure 3). The height of the trapping region is approximately equal to the diameter of a single yeast cell. Because the cells were immobilized in the device, the surrounding media could be changed as fast as necessary without the risk of dilution. This allowed us to achieve medium switching within a $1 \mathrm{~s}$ interval. Once the cell traps are completely full, the division of any cell causes the release of another cell into the media flow and the eventual exit of that cell into the effluent. Therefore, although the culture inside the device is maintained in log-phase, the density of the culture is constant, and the population of cells in the effluent at any time should be an accurate representation of the population in the cell traps.

Our computational model predicted that the final ratio of WT cells in a mixed population depends on the total number of glucose switches that have occurred, but not on the frequency of the switches. WT cells are predicted to experience a growth advantage during the first division in a glucose-rich environment. To test these predictions, we performed a series of experiments where a mixed population was exposed to square wave oscillations of galactose and glucose with various periods. The shortest period length was determined by the generation time of a yeast cell $(\approx 2 \mathrm{~h})$ and set at $4 \mathrm{~h}(2 \mathrm{~h}$ galactose, $2 \mathrm{~h}$ glucose). Since cells have galactose memory for around six division cycles, the total time spent in glucose was limited to $\leq 9$ h. ${ }^{14}$ We did not intend to study the effects of galactose memory loss, so we chose our longest switching period to be $10 \mathrm{~h}(5 \mathrm{~h}$ galactose, $5 \mathrm{~h}$ glucose).

To control for any effects in long-term growth in either of the carbon sources, we measured the WT:ST ratio of cultures experiencing growth in only galactose or only glucose. The change in the population composition under these static conditions was minimal, with a change of $-7 \%$ in the WT fraction occurring over 19 days in glucose medium. Growth in a constant galactose environment produced a $5 \%$ increase in the WT fraction over the same 19 days (Figure 5A). In contrast, carbon source fluctuations with a $10 \mathrm{~h}$ period lead to a $22 \%$ increase in WT over 19 days. Therefore, the large changes in population ratios observed in dynamic conditions are due to media switching and not to a competitive difference between the strains in either medium.

For all periods of sugar switching, the WT strain gradually took over the population of the culture. The rate of change varied with the frequency of media switching (Figure 5B). To achieve a comparable number of switches with different driving frequencies, the total length of the experiments ranged from 12 days $(T=4 \mathrm{~h})$ to 25 days $(T=8 \mathrm{~h})$. The $4 \mathrm{~h}$ period resulted in the fastest rate of population change, reaching WT > $90 \%$ within 12 days. Doubling the period of the media oscillation nearly doubled the time required for the WT to take over, with the $8 \mathrm{~h}$ period experiments reaching WT $>90 \%$ in 24 days, while the $10 \mathrm{~h}$ cycling cultures achieved only WT $>75 \%$ in 22 


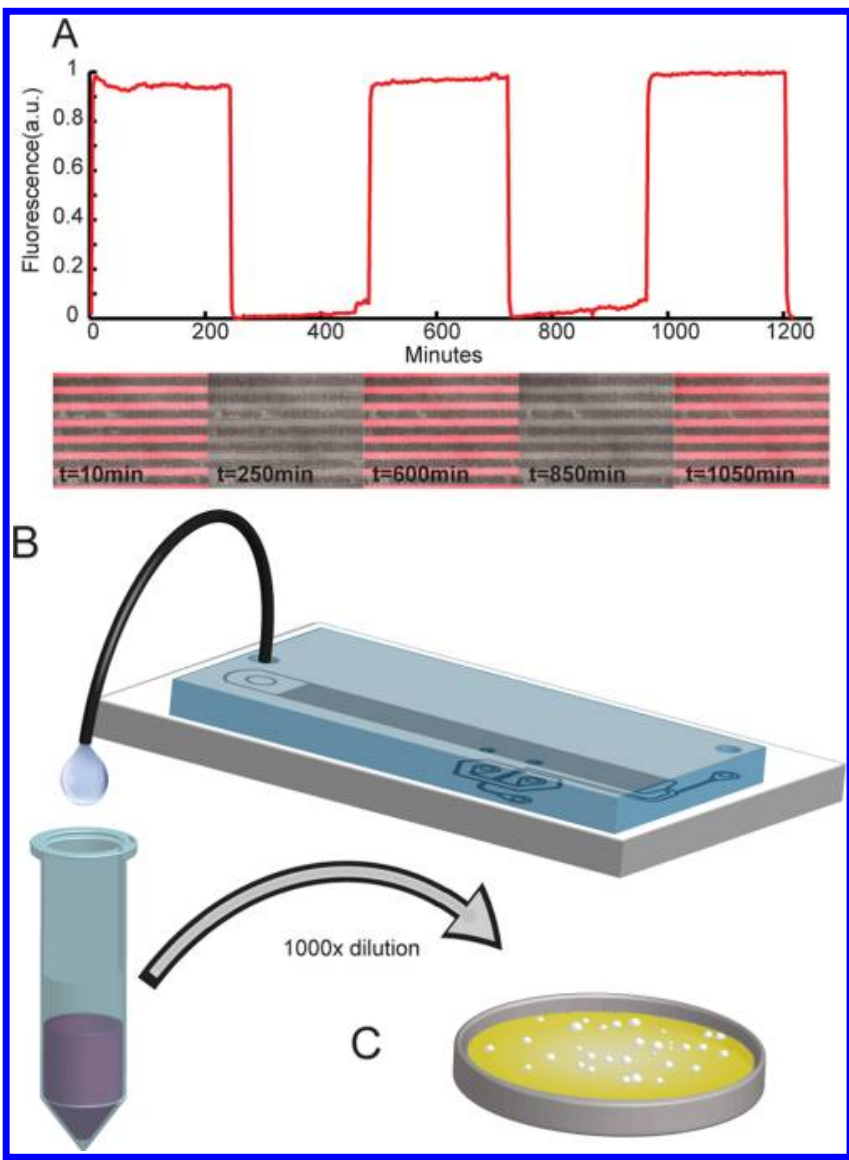

Figure 4. Overview of the experimental procedure. (A) Square wave of $0.25 \%$ glucose with different periods pulsed over a background of $0.5 \%$ galactose synthetic complete media. Tracer dye was added to the glucose media during testing and experiments to confirm proper function of the device. Pressure changes for media switching were accomplished off-chip using the custom built device described in the Supporting Information. Cell growth and function of media switching was confirmed using fluorescent imaging. Sample images show that traps remain full throughout the entire experiment. (B) Waste from the device, carrying population samples, was collected in a tube over the period of $24 \mathrm{~h}$. (C) The samples were serially diluted 1:1000 and plated on -Trp (ST) and -Leu (WT) plates. Colonies were visible after $48 \mathrm{~h}$ incubation at $30^{\circ} \mathrm{C}$. Plates were imaged, and the total number of colonies on each plate was determined.

days. Consistent with our model predictions, when the population ratio is plotted as a function of the number of carbon source switches, the results from all conditions fall on the same curve (Figure 5B). We calculated that the total number of switches required to reach WT $>85 \%$ is 60 for 4 and $8 \mathrm{~h}$ periods. Due to the length of the experiments with a $10 \mathrm{~h}$ period, the population did not exceed $75 \% \mathrm{WT}$; however, the trend in these experiments is highly similar to the results from shorter cycling periods. Statistical analysis of the slopes obtained from linear fits of the population ratios versus time for each period indicated that all three cycling periods produced statistically different population dynamics (z-test with $p=0.05$ ). The same statistical test of the slopes of the population ratios versus the number of switches showed that the selective effect of each carbon source switch did not vary statistically with cycling period.

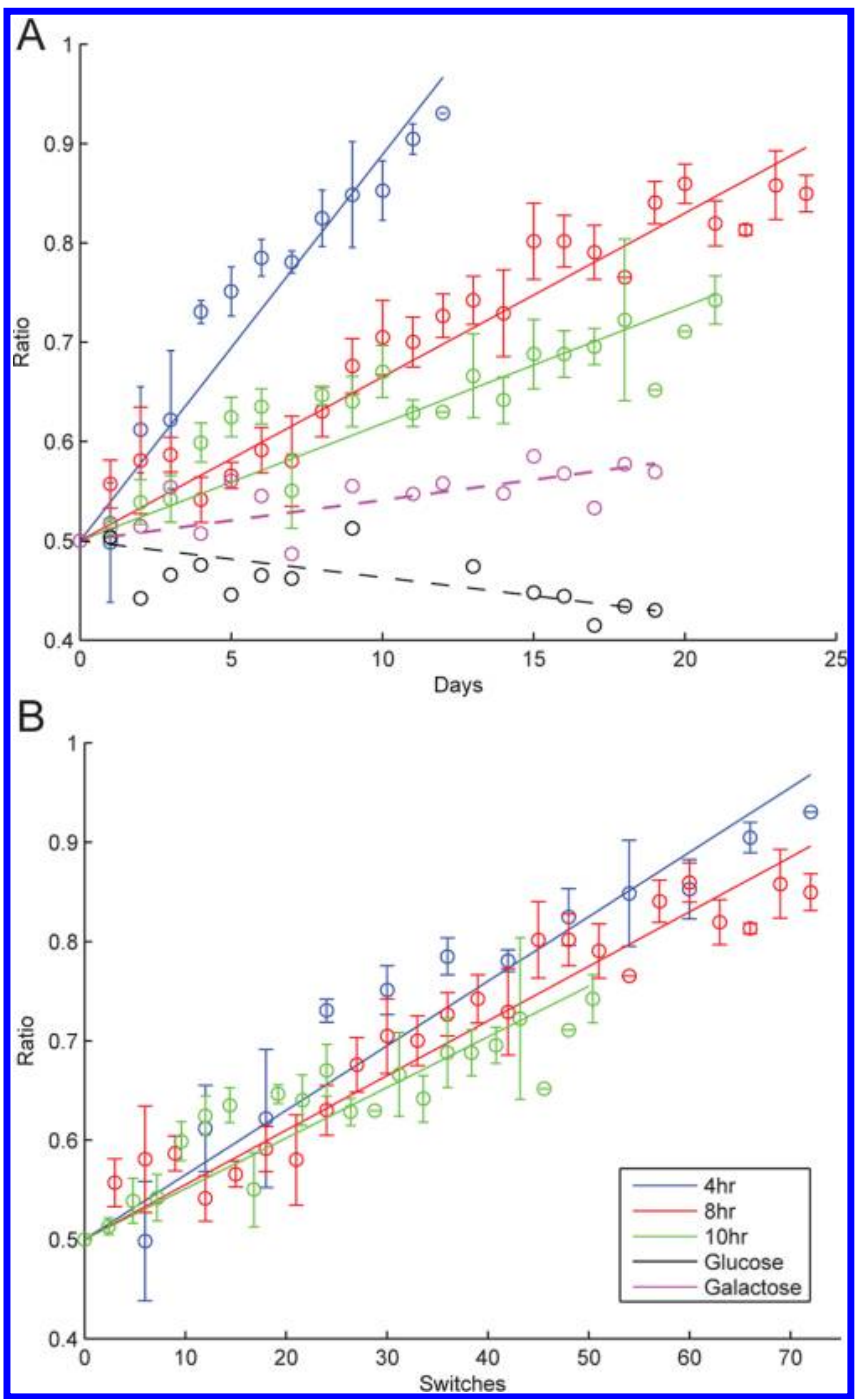

Figure 5. Experimental results of driving WT and ST mixed populations with different periods of galactose/glucose. (A) A mixed population of WT and ST cells were grown in constant $0.5 \%$ galactose background with square wave of $0.25 \%$ glucose pulsed at different periods. Samples were collected at the exit port of the microfluidic device and plated on auxotrophic plates. The ratio of WT to total number of cells for each experiment was scaled to start at a common value of $50 \%$. Actual starting ratios were with $10 \%$ of the common value. Glucose and galactose data point represent experiments in constant mediums. (B) The ratios were plotted vs calculated number of switches for each period. Error bars represent standard error.

\section{DISCUSSION}

Cells growing in a constant environment, such as a laboratory culture, are free to dedicate all of their resources to growth and division and often reach their maximal growth rate. However, in the natural world, cells rarely experience static conditions. Whether they exist as isolated cells or as part of a large multicellular organism, natural-living cells must cope with frequent changes to their surroundings. To survive in a dynamic environment, cells are equipped with gene networks that allow growth to continue in spite of changing conditions. However, this flexibility comes at a price, and cells experiencing environmental fluctuations usually do not attain their fastest growth rate. In light of this, it is likely that there are genetic mechanisms that exist because they have been selected for in natural environments, though they appear to have little 
competitive advantage in highly controlled laboratory experiments. In this study, we measured the adaptive advantage of one such mechanism that allows cells to respond rapidly to changes in glucose availability. As illustrated in Figure 1, cells quickly shorten the time spent between cell divisions when glucose is introduced into the environment. Interestingly, this mechanism is only active in cells that have experienced glucose in their recent past. Cells that have only previously been grown in galactose do not respond quickly to glucose, but have shorter cell division times in galactose than those with glucose experience. Therefore, in a dynamic environment, cells trade slower growth in the absence of glucose for the ability to rapidly respond to its return. This can be thought of as a history-dependent form of bet-hedging, whereby a cell anticipates the presence of its favorite carbon source for a while after its removal, but that anticipation fades over time if glucose does not return. ${ }^{15-18}$ Similarly, mechanisms involving chromatin organization and the transcription of GAL genes exist to provide cells with galactose memory. ${ }^{19}$

Glucose-sensitive transcripts have been described as part of several glucose-repressed pathways, suggesting that this may be a conserved mechanism for regulating the response to transient glucose availability in general, not just in the presence of galactose. This further suggests that glucose memory conveys a significant selective advantage to cells growing in dynamic environments. Consistently, we have observed that ST colonies do not grow well, relative to their WT counterparts, when galactose and glucose alternate continuously in the environment. However, whether the competitive translation mechanism is sufficient to explain the advantage of the WT genotype was less clear, as the resulting difference in cell division dynamics between WT and ST is small and transient. Our computational model was designed to specifically answer this question. In the model, the cell doubling time is dependent on the level of GAL1 mRNA. Therefore, differences in growth rate between WT and ST are directly related to the half-lives of GAL1 transcripts in the two strains, which differ in the presence of glucose. Our simulations, as well as our experimental results, confirm that glucose-mediated degradation of GAL1 transcripts provides a sufficient growth advantage to be selected for in dynamic environments. These results are important in that they illustrate the selective power of environmental fluctuations on seemingly modest phenotypic differences in cellular response dynamics.

A general interest in how life evolved has been at the center of biological study for more than a century. Maps of the evolutionary trajectories of species that are currently inhabiting the planet, and hypotheses of how others have become extinct are always rooted in speculation. To meet the challenges that go along with retroactively interpreting evolutionary mechanisms, researchers set up forward evolution experiments using microbes to measure the competitive fitness of particular genotypes in predetermined environments. Combined with the power of genomics technology, this method for determining the fitness associated with particular genetic variations is potentially revolutionary. However, genome-wide measurements of competitive fitness using the yeast deletion collection have yielded only moderate results that have called into question long held theories explaining natural selection. ${ }^{20-26}$ Particularly bewildering has been the discovery that only 20\% of yeast genes are essential, and most gene deletions have little to no apparent effect on competitive fitness. ${ }^{27,28} \mathrm{How}$, then, is genetic integrity maintained if the majority of the genome is dispensable? The answer could be that the fitness contribution of individual genes is dependent on interactions with a dynamic environment in which yeast cells are evolved. ${ }^{29-34}$ As we show in this study, the relationship between genes and fitness is potentially highly nuanced, incorporating changing conditions in the present as well as information about past growth environments and the anticipation of future events.

We invented our evoDAW device to measure fitness advantages that are strongly dependent on environmental dynamics. To test the device, we set up a competition experiment between two strains with a predicted fitness difference in environments that were known to favor the growth of one strain over the other. The resulting population dynamics that we measured were in good agreement with theoretical predictions for each environment, and were highly reproducible relative to competition experiments carried out in batch cultures. The reproducibility of our results may be attributed to the multiple cell traps (10 total) that each evoDAW device contains. Any variation due to uneven loading of the two strains is likely to have been normalized because each device essentially contains 10 individual populations. We tracked the evolution of the population by selecting for metabolic markers that differed between the two strains. However, the amount of cells collected each day $\left(\sim 10^{7}\right)$ is sufficient for use in many cellular and molecular assays, such as immunoblotting, flow cytometry, and qPCR. If more material were desired, for use in genomic techniques for example, the collected cells could be amplified through growth in rich medium. In this study, experiments were performed for $\sim 500$ generations or $\sim 30$ days, but there is no theoretical limit to how long these cultures can be maintained. Finally, the dimensions of the growth chambers can easily be altered to accommodate microbes other than yeast or animal cell cultures. The evoDAW chip provides a powerful platform to measure the fitness contributions of individual genes in dynamic conditions that more accurately reflect the environments in which cells have evolved.

\section{CONCLUSIONS}

In conclusion, we have shown that a microfluidic chemostat can be used to study population dynamics in the presence of precisely controlled environmental fluctuations. The results of our modeling and experiments support the conclusion that environmental dynamics can contribute to the selection of particular traits, even when those traits do not lead to measurable gains in fitness during growth in static environments. In future studies, this experimental platform may be used to determine the environmental conditions that have naturally selected for the large number of yeast genes that do not contribute a fitness advantage in standard laboratory evolution experiments.

\section{ASSOCIATED CONTENT}

\section{S Supporting Information}

Detailed descriptions of design criteria used to build and test the evoDAW device are included in the SI. Also included is information on the derivation of the mathematical model for growth in dynamic environments, the numerical simulations of population dynamics in those environments, and definitions of variables used in mathematical equations. This material is available free of charge via the Internet at http://pubs.acs.org. 


\section{AUTHOR INFORMATION}

\section{Corresponding Author}

*E-mail: jhasty@ucsd.edu.

\section{Author Contributions}

${ }^{\nabla}$ Contributed equally to this work.

Notes

The authors declare no competing financial interest.

\section{ACKNOWLEDGMENTS}

This work was supported by the National Science Foundation (MCB-1121748) and by the San Diego Center for Systems Biology (NIH Grant P50 GM085764). We gratefully acknowledge Dr. Michael Ferry for helpful discussions and advice with microfluidics designs. M.R.B. is supported by the National Institutes of Health through the Joint NSF/NIGMS Mathematical Biology Program, Grant Number R01GM104974, and the Welch Foundation, Grant Number C-1729.

\section{REFERENCES}

(1) Darwin, C. On the Origins of Species by Means of Natural Selection; Murray: London, 1859.

(2) Bennett, M. R.; Hasty, J. Microfluidic Devices for Measuring Gene Network Dynamics in Single Cells. Nat. Rev. Genet. 2009, 10, 628-638.

(3) Balagaddé, F. K.; You, L.; Hansen, C. L.; Arnold, F. H.; Quake, S. R. Long-Term Monitoring of Bacteria Undergoing Programmed Population Control in a Microchemostat. Science 2005, 309, 137-140.

(4) Balaban, N. Q.; Merrin, J.; Chait, R.; Kowalik, L.; Leibler, S. Bacterial Persistence as a Phenotypic Switch. Science 2004, 305, 16221625 .

(5) Gottschamel, J.; Richter, L.; Mak, A.; Jungreuthmayer, C.; Birnbaumer, G.; Milnera, M.; Bruckl, H.; Ertl, P. Development of a Disposable Microfluidic Biochip for Multiparameter Cell Population Measurements. Anal. Chem. 2009, 81, 8503-8512.

(6) Ferry, M. S.; Razinkov, I. A. Microfluidics for Synthetic Biology: From Design to Execution. Methods Enzymol. 2011, 497, 295-372.

(7) Bennett, M. R.; Pang, W. L.; Ostroff, N. A.; Baumgartner, B. L.; Nayak, S.; Tsimring, L. S.; Hasty, J. Metabolic Gene Regulation in a Dynamically Changing Environment. Nature 2008, 454, 1119-1122.

(8) Lohr, D.; Venkov, P.; Zlatanova, J. Transcriptional Regulation in the Yeast GAL Gene Family: A Complex Genetic Network. FASEB J. 1995, 9, 777-787.

(9) Johnston, G.; Singer, R.; Sharrow, S.; Slater, M. Cell Division in the Yeast Saccharomyces cerevisiae Growing at Different Rates. J. Gen. Microbiol. 1980, 118, 479-484.

(10) Scheffler, I. E.; de la Cruz, B. J.; Prieto, S. Control of mRNA Turnover as a Mechanism of Glucose Repression in Saccharomyces cerevisiae. Int. J. Biochem. Cell Biol. 1998, 30, 1175-1193.

(11) Andrade, R. P.; Kötter, P.; Entian, K.-D.; Casal, M. Multiple Transcripts Regulate Glucose-Triggered mRNA Decay of the Lactate Transporter JEN1from Saccharomyces cerevisiae. Biochem. Biophys. Res. Commun. 2005, 332, 254-262.

(12) Baumgartner, B. L.; Bennett, M. R.; Ferry, M.; Johnson, T. L.; Tsimring, L. S.; Hasty, J. Antagonistic Gene Transcripts Regulate Adaptation to New Growth Environments. Proc. Natl. Acad. Sci. U.S.A. 2011, 108, 21087-21092.

(13) Zhang, Z.; Boccazzi, P.; Choi, H.-G.; Perozziello, G.; Sinskey, A. J.; Jensen, K. F. Microchemostat-Microbial Continous Culture in a Polymer-Based, Instrumented Microbioreactor. Lab Chip 2006, 6, 906-913.

(14) Brickner, D. G.; Cajigas, I.; Fondufe-Mittendorf, Y.; Ahmed, S.; Lee, P.-C.; Widom, J.; Brickner, J. H. H2A.Z-Mediated Localization of Genes at the Nuclear Periphery Confers Epigenetic Memory of Previous Transcriptional State. PLoS Biol. 2007, 5, 704-716.
(15) Levy, S. F.; Ziv, N.; Siegal, M. L. Bet Hedging in Yeast by Heterogeneous, Age-Correlated Expression of a Stress Protectant. PLoS Biol. 2012, 10, e1001325.

(16) Veening, J.-W.; Stewart, E. J.; Berngruber, T. W.; Taddei, F.; Kuipers, O. P.; Hamoen, L. W. Bet-Hedging and Epigenetic Inheritance in Bacterial Cell Development. Proc. Natl. Acad. Sci. U.S.A. 2008, 105, 4393-4398.

(17) Beaumont, H. J.; Gallie, J.; Kost, C.; Ferguson, G. C.; Rainey, P. B. Experimental Evolution of Bet Hedging. Nature 2009, 462, 90-93.

(18) Nevozhay, D.; Adams, R.; Van Itallie, E.; Bennett, M. R.; Balazsi, G. Mapping the Environmental Fitness Landscape of a Synthetic Gene Circuit. PLoS Comput. Biol. 2012, 8, e1002480.

(19) Zacharioudakis, I.; Gligoris, T.; Tzamarias, D. A Yeast Catabolic Enzyme Controls Transcriptional Memory. Curr. Biol. 2007, 17, 2041-2046.

(20) Fisher, R. The Theory of Natural Selection; Oxford University Press: London, 1930.

(21) Giaever, G.; Chu, A. M.; Ni, L.; Connelly, C.; Riles, L.; Veronneau, S.; Dow, S.; Lucau-Danila, A.; Anderson, K.; Andre, B. Functional Profiling of the Saccharomyces cerevisiae Genome. Nature 2002, 418, 387-391.

(22) Steinmetz, L. M.; Sinha, H.; Richards, D. R.; Spiegelman, J. I.; Oefner, P. J.; McCusker, J. H.; Davis, R. W. Dissecting the Architecture of a Quantitative Trait Locus in Yeast. Nature 2002, 416, 326-330.

(23) Deutschbauer, A. M.; Jaramillo, D. F.; Proctor, M.; Kumm, J.; Hillenmeyer, M. E.; Davis, R. W.; Nislow, C.; Giaever, G. Mechanisms of Haploinsufficiency Revealed by Genome-Wide Profiling in Yeast. Genetics 2005, 169, 1915-1925.

(24) Sliwa, P.; Korona, R. Loss of Dispensable Genes is Not Adaptive in Yeast. Proc. Natl. Acad. Sci. U.S.A. 2005, 102, 17670-17674.

(25) Breslow, D. K.; Cameron, D. M.; Collins, S. R.; Schuldiner, M.; Stewart-Ornstein, J.; Newman, H. W.; Braun, S.; Madhani, H. D.; Krogan, N. J.; Weissman, J. S. A Comprehensive Strategy Enabling High-Resolution Functional Analysis of the Yeast Genome. Nat. Methods 2008, 5, 711-718.

(26) Jasnos, L.; Tomala, K.; Paczesniak, D.; Korona, R. Interactions Between Stressful Environment and Gene Deletions Alleviate the Expected Average Loss of Fitness in Yeast. Genetics 2008, 178, 21052111.

(27) Thatcher, J.; Shaw, J. M.; Dickinson, W. Marginal Fitness Contributions of Nonessential Genes in Yeast. Proc. Natl. Acad. Sci. U.S.A. 1998, 95, 253-257.

(28) Bell, G. Experimental Genomics of Fitness in Yeast. Proc. R. Soc. B 2010, 277, 1459-1467.

(29) Brown, J. A.; Sherlock, G.; Myers, C. L.; Burrows, N. M.; Deng, C.; Wu, H. I.; Mc- Cann, K. E.; Troyanskaya, O. G.; Brown, J. M. Global Analysis of Gene Function in Yeast by Quantitative Phenotypic Profiling. Mol. Syst. Biol. 2006, 2, 1-9.

(30) Parsons, A. B.; Lopez, A.; Givoni, I. E.; Williams, D. E.; Gray, C. A.; Porter, J.; Chua, G.; Sopko, R.; Brost, R. L.; Ho, C.-H. Exploring the Mode-of-Action of Bioactive Compounds by Chemical-Genetic Profiling in Yeast. Cell 2006, 126, 611-625.

(31) Williams, P. D.; Hastings, A. Paradoxical Persistence Through Mixed-System Dynamics: Towards a Unified Perspective of Reversal Behaviours in Evolutionary Ecology. Proc. R. Soc. B 2011, 278, 12811290.

(32) Wolf, D. M.; Vazirani, V. V.; Arkin, A. P. Diversity in Times of Adversity: Probabilistic Strategies in Microbial Survival Games. J. Theor. Biol. 2005, 234, 227-253.

(33) Acar, M.; Mettetal, J. T.; van Oudenaarden, A. Stochastic Switching as a Survival Strategy in Fluctuating Environments. Nat. Genet. 2008, 40, 471-475.

(34) Kussell, E.; Leibler, S. Phenotypic Diversity, Population Growth, and Information in Fluctuating Environments. Science 2005, 309, 2075-2078. 